\title{
Mechanism of plant adaptation to changing illumination by rearrangements of their photosynthetic apparatus
}

\author{
V.I. Belkov*, E.Yu. Garnik, Yu.M. Konstantinov \\ Siberian Institute of Plant Physiology and Biochemistry, Siberian Branch of the Russian Academy of Sciences, Irkutsk, Russia
}

DOI 10.18699/ICG-PlantGen2019-32

(c) Autors, 2019

* e-mail: anvad.irk@rambler.ru

\begin{abstract}
In this review, the mechanism and the physiology of the state transition process are overviewed. This mechanism allows plants to adapt their photosynthetic apparatus to changes in light conditions. State transitions are possible due to the specific structure of the photosystem II external light-harvesting antenna, which is able to move between photosystems II and I. As a result, the electron flow rate in the electron-transport chain of chloroplasts changes. The structure of photosystems II and I and the composition of photosystem II supercomplexes are described.

Key words: plant photosystems; PSI; PSII; chloroplast proteins; illuminance.
\end{abstract}

\section{Introduction}

Light is the most important environmental factor for photosynthetic organisms. This is a variable and unstable factor. For this reason, plants have developed appropriate adaptation mechanisms. There are short-term and long-term responses occurring in plants under shifts of illuminance quality and intensity (Dietzel et al., 2008). Short-term responses (a few seconds or minutes) induce lumen protonation and phosphorylation of various chloroplast proteins. These responses lead to non-photochemical quenching and the state transitions process. Long-term responses emerge after relatively long periods of time (hours or days, or during seasonal changes) and lead to sustainable changes in the content of chloroplast components (chlorophyll $a / b$ ratio, photosystem proteins and light-harvesting complexes) through changes in the expression of the corresponding genes (Dietzel et al., 2015).

Light reactions in plant chloroplasts are provided by four major complexes located in the thylakoid membrane: photosystem I (PSI), photosystem II (PSII), cytb6/f complex and ATP synthase. The understanding of the state transition mechanism is related to the study of the structure of the external antenna of PSII, which is represented by proteins of light-harvesting complex II (LHCII). A part of the external PSII antenna is mobile and can move between two photosystems. This mobility is the basis of the state transitions process. Thus, the state transitions process is one of ways to adapt to light fluctuations (Caffarri et al., 2015).

\section{Structure of photosystems in plants}

PSII is mostly located in grana stacks, whereas PSI is located in stroma lamellae (Albertsson, 2001; Dekker, Boekema, 2005). Both photosystems have similar structures and consist of two parts, a core protein complex and a light-harvesting antenna (light-harvesting complex I (LHCI) for PSI and lightharvesting complex II (LHCII) for PSII, respectively). The core complex is mostly represented by conservative proteins, which are largely similar in prokaryotes and eukaryotes except for small differences - the sites of photochemical reactions. In contrast, the structure of light-harvesting antennas (especially external antennas) in different photosynthetic organisms is not the same. External antenna proteins are responsible for light absorption and regulation of the photosynthetic processes (Horton et al., 1996).

The reaction center (RC) of PSII is a heterodimer, composed of the PsbA (D1), PsbD (D2) proteins and associated with 6 chlorophyll $a$ and two pheophytins (Umena et al., 2011). There are many small protein subunits of the Psb family, which are associated with the RC. The core proteins of PSII are linked to the internal antenna (represented by the CP47 (PsbB) and CP43 (PsbC) proteins, which are associated with 16 and 13 chlorophyll $a$ molecules, respectively) and a number of small subunits (Shi et al., 2012). The association of the PsbA, PsbD, $\mathrm{PsbB}$ and $\mathrm{PsbC}$ proteins in higher plants is called the Core of PSII (C). This core is connected with a system of external antennas, which consists of light-harvesting complex proteins associated with chlorophylls $a / b$ and are represented by the Lhcb1-6 subunits. This external antenna complex consists of trimers of Lhcb1-3 subunits, which are associated with the core complex by the minor subunits of Lhcb4-6 (Gao et al., 2018). The union of the core with the external LHC antenna is called the 'PSII supercomplex'.

It should be noted that the qualitative composition of the PSII supercomplex is not constant, and depends on the lightharvesting antenna size. The PSII supercomplex includes $\mathrm{C}$ and strongly (S), moderately (M) and loosely (L) bound LHCII trimers. LHCII-S connection is provided by association of LHCII proteins with PsbC and Lhcb5; LHCII-M connection, by the Lhcb6 and Lhcb4 proteins, which are located on a part of the PsbB protein. There are different variations of supercomplexes formed in thylakoid membranes $\left(\mathrm{C}_{2} \mathrm{~S}, \mathrm{C}_{2} \mathrm{M}\right.$, $\mathrm{C}_{2} \mathrm{~S}_{2}, \mathrm{C}_{2} \mathrm{SM}, \mathrm{C}_{2} \mathrm{~S}_{2} \mathrm{M}, \mathrm{C}_{2} \mathrm{~S}_{2} \mathrm{M}_{2}$ and the $\mathrm{C}_{2} \mathrm{~S}_{2} \mathrm{M}_{2} \mathrm{~L}_{2}$ megacomplex) (Nosek et al., 2017). $\mathrm{C}_{2} \mathrm{~S}$ is the smallest supercomplex, which contains the core dimer of PSII, the Lhcb4 and Lhcb5 proteins and one LHCII trimer (Rantala et al., 2017). $\mathrm{C}_{2} \mathrm{~S}_{2} \mathrm{M}_{2}$ is the most common type of supercomplexes. In addition, the formation of $\mathrm{C}$ and CS complexes was also observed (Bielcznski et al., 2016). It was established that the external antenna of LHCII can migrate in the thylakoid membrane.

PSI consists of about 15 subunits. The RC of PSI is represented by the PsaA and PsaB proteins. The external antenna 
of PSI consists of four Lhca1-4 proteins, each of which is associated with $\sim 100$ molecules of chlorophylls $a / b$ and xanthophylls. The proteins of the external antenna extend around the RC of PSI. The structure of PSI included minor proteins (PsaC, PsaD, PsaE, PsaG, PsaK, PsaO, PsaH), which are involved in the docking with plastocyanine and ferredoxin, and also stabilize the LHCI antenna and the formation of a binding site with the LHCII protein. It is believed that the external antenna of LHCI is not able to move in the thylakoid membrane. In addition, PSI can be associated with the NADH dehydrogenase-like complex (NDH) resulting in the formation of a specific supercomplex involved in the cyclic electron flow (Yadav et al., 2017).

\section{The mechanism of state transitions}

State transitions is a mechanism of an absorbed light energy redistribution between two photosystems based on the reversible movement of LHCII trimers from PSII to PSI (Goldschmidt-Clermont, Bassi, 2015). According to this model, the excess of chlorophyll fluorescence is reduced (Bonaventura et al., 1969) and the electron flux of the electron-transport chain is stabilized (Dietzel et al., 2008). When PSII is overexcited, phosphorylation of LHCII (P-LHCII) occurs. These P-LHCII are dissociated from PSII and move from the stacks of thylakoids to lamellae where PSI is localized (State 2). As a result, the formation of state transitions supercomplex PSI-LHCI-LHCII occurs (Caffarri et al., 2014). When PSI is overexcited, P-LHCII is dephosphorylated by Protein Phosphatase1/Thylakoid-Associated Phosphatase PPH1/TAP38 and migrates back to PSII (State 1) (Kono et al., 2014). It was found that the LHCII proteins are phosphorylated in the N-terminal part. Hence, conformational changes of LHCII proteins occur and their affinity to PSII disappears (Nilson et al., 1997). Analysis of various mutants showed that the PsaH subunit of PSI is responsible for the physical placement of LHCII in PSI, and the PsaL, PsaO and PsaP subunits of PSI provide these interactions (Caffarri, 2015).

About $20 \%$ of LHCII proteins are involved in the transfer form PSII to PSI in Arabidopsis thaliana (Allen, 1992), while the figure for algae and cyanobacteria is up to $80 \%$, since this process is associated with switching between cyclic and linear electron transport (Goldschmidt-Clermont, Bass, 2015).

\section{Regulation of state transitions}

The state transition process is regulated through changes in the redox state of the plastoquinone pool. When PSII is excited, lastoquinone (PQ) reduces to plastoquinol (PQH2). $\mathrm{PQH} 2$ associates with a complex of cytb6/f and this interaction leads to activation of the protein kinase State Transition 7 (STN7). STN7 phosphorylates LHCII proteins (mostly Lhcb2 isoforms) and initiates state transition from State 1 to State 2 (Bellafiore et al., 2005; Longoni et al., 2017). This kinase functions as a dimer associated with the cytb6/f complex (Wunder et al., 2013). STN7 kinase has a transmembrane domain, which is located in the N-terminal part (on the lumen side), and a catalytic domain located on the stroma side of the thylakoid membrane.

In 2011, a model of STN7 regulation by ferredoxin/thioredoxin was proposed (Puthiyveetil, 2011). According to this model, when PSII is excited, $\mathrm{PQH} 2$ restores the disulfide bond in STN7 cysteines located on the lumen side and activates STN7. In high-light conditions, STN7 is inhibited by ferredoxin/thioredoxin signals.

\section{Conclusion}

At present, the structure of the PSII supercomplexes, the composition and location of their subunits are under study. The formation of these supercomplexes leads to the efficient usage of absorbed light energy. State transitions lead to the reversible movement of LHCII proteins between both photosystems. It is a subtle adaptation mechanism that allows plants to respond to changes in light conditions. In the evolutionary context, this mechanism was formed in cyanobacteria, algae and higher plants. It was established that the regulation of this process depends on the rate of electron flow in the electrontransport chain as well as protein kinases and phosphatases activity. Thus, the study of the processes of regulation of protein kinases and phosphatases can contribute to the further study of plant adaptation mechanisms.

\section{References}

Umena Y., Kawakami K., Shen J.-R., Kamiya N. Crystal structure of oxygen-evolving photosystem II at a resolution of $1.9 \AA$. Nature. 2011;473:55-60.

Horton P., Ruban A.V., Walters R.G. Regulation of Light harvesting in Green Plants. Annu. Rev. Plant Physiol. Plant Mol. Biol. 1996; 47:655-684.

Nosek L., Semchonok D., Boekema E.J., Ilik P., Kouril R. Structural variability of plant photosystem II megacomplexes in thylakoid membranes. Plant Journal. 2017;89:104-111.

Gao J., Wang H., Yuan Q., Feng Y. Structure and Function of the Photosystem Supercomplexes. Frontiers Plant Sci. 2018;9 (Article 357): $1-7$.

Allen J.F. Protein phosphorylation in regulation of photosynthesis. Biochim. Biophys. Acta-Bioenerg. 1992;1098:275-335.

Albertsson P. A quantitative model of the domain structure of the photosynthetic membrane. Trends Plant Sci. 2001;6:349-354. DOI 10.1016/S1360-1385(01)02021-0.

Dekke J.P., Boekema E.J. Supramolecular organization of thylakoid membrane proteins in green plants. Biochim. Biophys. Acta. 2005; 1706:12-39. DOI 10.1016/j.bbabio.2004.09.009.

Bellafiore S., Barneche F., Peltier G., Rochaix J.D. State transitions and light adaptation require chloroplast thylakoid protein kinase STN7. Nature. 2005;433:892-895. DOI 10.1038/nature03286.

Longoni P., Douchi D., Cariti F., Fucile G., Goldschmidt-Clermont M. Phosphorylation of the Light-Harvesting Complex II Isoform Lhcb2 Is Central to State Transitions. Plant Physiol. 2015;169:2874-288.

Wunder T., Liu Q., Aseeva E., Bonardi V., Leister D., Pribil M. Control of STN7 transcript abundance and transient STN7 dimerisation are involved in the regulation of STN7 activity. Planta. 2013;237: $541-558$.

Puthiyaveetil S. A mechanism for regulation of chloroplast LHC II kinase by plastoquinol and thioredoxin. FEBS Lett. 2011;585:17171721.

Dietzel L., Brautigam K., Pfannschmidt T. Photosynthetic acclimation: state transitions and adjustment of photosystem stoichiometry-functional relationships between short-term and long-term light quality acclimation in plants. FEBS J. 2008;275:1080-1088.

Dietzel L., Glaber C., Liebers M., Hiekel S., Courtois F., Czarnecki O., Schlicke H., Zubo Y., Borner T., Mayer K., Grimm B., Pfannschmidt $\mathrm{T}$. Identification of early nuclear target genes of plastidial redox signals that trigger the long-term response of Arabidopsis to light quality shifts. Mol. Plant. 2015;8:1237-1252. 
Eberhard S., Finazzi G., Wollman F.A. The dynamics of photosynthesis. Annu. Rev. Genet. 2008;42:463-515.

Caffarri S., Tibiletti T., Jennings R.C., Santabarbara S. A Comparison Between Plant Photosystem I and Photosystem II Architecture and Functioning. Current Protein Peptide Sci. 2014;15:296-331.

Shi L.-X., Hall M., Funk C., Schrцder W.P. Photosystem II, a growing complex: updates on newly discovered components and low molecular mass proteins. Biochim. Biophys. Acta. 2012;1817:13-25. DOI 10.1016/j.bbabio.2011.08.008.

Rantala M., Tikkanen M., Aro E.-M. Proteomic characterization of hierarchical megacomplex formation in Arabidopsis thylakoid membrane. Plant Journal. 2017;92:951-962.

Bielczynski L.W., Schansker G., Croce R. Effect of Light Acclimation on the Organization of Photosystem II Super- and Sub-Complexes in Arabidopsis thaliana. Frontiers Plant Sci. 2016;7. Article 105.

Yadav K.N., Semchonok D.A., Nosek L., Kouřil R., Fucile G., Boekema E.J., Eichacke L.A. Supercomplexes of plant photosystem I with cytochrome b6f, light-harvesting complex II and NDH. Biochim. Biophys. Acta. 2017;1858:12-20.

Bonaventura C., Myers J. Fluorescence and oxygen evolution from Chlorella pyrenoidosa. Biochim. Biophys. Acta 1969;189:366-383.

Goldschmidt-Clermont M., Bassi R. Sharing light between two photosystems: mechanism of state transitions. Current Opinion Plant Biol. 2015;25:71-78.

Kono M., Terashima I. Long-term and short-term responses of the photosynthetic electron transport to fluctuating light. J. Photochemistry Photobiol. 2014;137:89-99.

Nilsson A., Stys D., Drakenberg T., Spangfort M.D., Forsen S., Allen J.F. Phosphorylation controls the three-dimensional structure of plant light harvesting complex II. J. Biol. Chem. 1997;272:18350-18357.

Anknowlegments. This work was supported by the RFBR No. 1834-00800.

Conflict of interest. The authors declare no conflict of interest. 